\title{
Global left ventricular function assessment by ECG-gated multi-detector CT (MDCT): revised role in relation to $2 \mathrm{D}$ transthoracic echocardiography
}

\author{
Mohammed Elsayed Abdel Samea ${ }^{1}$, Ashraf Anas Zytoon ${ }^{2^{*}}$ (D), Asmaa Mohamed Abd Elaziz Abo Mostafa ${ }^{2}$ and \\ Shaimaa Abdel-Hamid Hassanein ${ }^{2}$
}

\begin{abstract}
Background: An accurate and reproducible way for determining the left ventricular function is crucial to provide diagnostic and prognostic aspects of the pump activity of the heart. The MDCT of the heart can be that modality. We compared the $128 \mathrm{MDCT}$ of fifty patients with their 2D echocardiography performed on the same day.

Results: Mean EF, ESV, EDV, and LV mass were $61.22 \pm 9.50 \%, 70.23 \pm 38.35,172.22 \pm 53.57,164.63 \pm 52.57$ respectively on MDCT, and $61.14 \pm 10.90 \%, 72.13 \pm 32.69,173.76 \pm 62.45,198.32 \pm 72.54$ respectively on echocardiography with moderate correlation in $\mathrm{EF}$ and good correlation in ventricular volumes $(p<0.05)$ using linear regression analysis. A Bland-Altman analysis showed that MDCT had slightly lower LFEF, LVESV, and LVEDV values with mean value of differences of $0.8,2.4$, and 2.28 respectively.

Conclusion: It is reasonable to use MDCT alone to assess LV function in patients already underwent coronary CT angiography.
\end{abstract}

Keywords: MDCT, Left ventricular systolic function, 2D echocardiography

\section{Background}

The evaluation of cardiac function can provide important diagnostic and prognostic information in many diseases that affect the pump activity of the heart [1]. Transthoracic echocardiography is the most common modality doctors used for evaluation of LV function, but it is operator dependent and it is also impaired by a poor acoustic window [2]. Nowadays cardiac CT is used to evaluate the coronary artery diseases. Measurement of the left ventricular function can be performed by cardiac CT [3]. The acquisition of the entire heart volume in a single breath-hold can be done by MDCT with good temporal and spatial resolution. Moreover, information

\footnotetext{
* Correspondence: ashradio@gmail.com

${ }^{2}$ Radiodiagnosis Department, Faculty of Medicine, Menoufia University, Menoufia, Egypt

Full list of author information is available at the end of the article
}

for any phase of the cardiac cycle can be taken from data acquisition in spiral MDCT with retrospective ECG gating, so, end-systolic and end-diastolic images can be used to measure ventricular volumes and function [4]. The aim of the current study was the evaluation of the role of multi-detector-row computed tomography (MDCT) in assessing left ventricular systolic function compared with transthoracic echocardiography.

\section{Methods \\ Study population}

This study was performed on 50 patients (27 of them were male and 23 were female) with a mean age of $50.36 \pm$ 10.24 years. They were presented with atypical chest pain, dyspnea, or regular arrhythmia. All patients were referred to the Radiology Department at our institute. All patients 
were scanned on 128 slice MDCT and underwent 2D echocardiography on the same day of the CT scan.

We followed all applicable institutional regulations during the data collection of this study to achieve the ethical use of human individuals. Participants assured that the data collected were handled confidentially before they gave their verbal consent to be included in the study. Institutional review board (IRB) study approval was obtained.

Exclusions criteria were absolute contraindication to radiation or contrast media like pregnancy, patients with relative contraindications such as atopy, asthma but was performed if the benefit of examination outweighed the risk in such patients, impaired renal function (serum creatinine level more than $1.5 \mathrm{mg} / \mathrm{dl}$ ), patients with arrhythmias and ectopic heartbeats were excluded as regular heart rate was required for CT coronary angiogram, unstable angina or acute coronary syndrome, and patients with a pacemaker and ventricular septal defect were also excluded because successful segmentation of the LV blood pool could not be done in these patients due to artifacts and incorrect segmentation.

\section{Patient preparation}

Before the examination, all patients undergoing CT coronary angiogram were assessed for the blood pressure and heart rate. In all patients with a resting heart rate exceeding 70 beats per minute, an oral dose of shortacting beta-blocker (propranolol $5 \mathrm{mg}$ ) was administered once daily 3 days before CT scan to make heart rate below 70 beats per minute. If still, heart rate is more than 70 beats per minute after 3 days preparation then a single dose of calmepam $(1.5 \mathrm{mg})$ and propranolol $5 \mathrm{mg}$ were given 1 hour before scan. In patients with contraindications for B-blockers, calcium channel blocker, or digitalis were used after consultation with their cardiologists.

\section{CCTA technique}

CCTA was performed on a 128-slice MDCT (Somatom definition AS 128 Siemens Healthineers, Erlangen, Germany) with a rotation time of $300 \mathrm{msec}$, matrix $256 \times$ 256, care Dose 4D (automated real-time tube current adjustment for best diagnostic image quality at lowest possible dose), The pitch was 0.18 and a slice collimation of $128 \times 0.6 \mathrm{~mm}$ using a continuous helical scan. The CT scan was automatically triggered by an automatic bolus tracking technique. A region of interest was placed into the proximal part of the descending aorta, and image acquisition began $4 \mathrm{~s}$ after the signal density level reached 100 Hounsfield units (HU). The contrast volume of 1.5 $\mathrm{mL} / \mathrm{kg}$ of with an average of $90 \mathrm{~mL}$ of non-ionic contrast media (Ultravist 370, BSP, Germany) was administered and this was followed by the administration of $20 \mathrm{~mL}$ of an 80 to $20 \%$ mix of Ultravist 370 and saline at a rate of $5.5 \mathrm{~mL}$ per second to increase the concentration of the contrast media in the left side of the heart and to facilitate segmentation of the right and left cardiac chambers. The patient position was feet first supine, and the image acquisition was craniocaudal for all patients.

The retrospective ECG-correlated image was reconstructed. For the assessment of LV function and volume, images were reconstructed at $10-100 \%$ of the R-R interval in $10 \%$ time increments. We used a slice thickness of $0.75 \mathrm{~mm}$ and a position increment of $0.5 \mathrm{~mm}$ with the field of view included the heart only to increase spatial resolution of the reconstructed images for the reconstruction of axial images obtained by CT scan. The reconstruction of these data was done along the entire cardiac cycle. Image data were evaluated with comprehensive cardiac analysis software on Philips Intellispace Portal v6.0.6.20039 workstation. The mitral valve plane was used by the software as an upper border for the left ventricle segmentation. The software identified the hinges of the mitral and aortic valve leaflets closest to the ventricle wall and selected them as defining points for the plane (Fig. 1a). Segmentation was performed during systole and diastole (Fig. 1b). Regarding this method, all CT scans were analyzed. So, it permitted optimal segmentation of the LV. Then papillary muscles were excluded from the cavity automatically. This led to the definite determination of blood volume in the LV. Then multiplanar reformats were performed by the software in short and long axes of heart. The long axis images were parallel to the interventricular septum and along with line connecting the middle level of the mitral valve and the LV apex. The short-axis images were performed parallel to the mitral valve plane. Once the region of interest was finished (checking the endocardial tracing was correct), the software calculated the EDV and ESV using the threshold-based method for estimation of left ventricular volumes (by differentiating the contrast filled lumen from the wall), and then the software calculated stroke volume (SV) and EF automatically from these values.

\section{ECHO examination technique}

Two-dimensional ECHO examination was performed within the same day of cardiac CT examination using Philips IU22 XMATRIX ultrasound system. All patients underwent 2D echocardiography using a standard protocol. Images were obtained using $(2.5-3.5 \mathrm{MHz})$ dedicated cardiac transducer. Patients were evaluated with two dimensional and M-mode echocardiographic examinations in the left lateral decubitus position, and images were acquired in standard echo views (four-chamber or fivechamber views and parasternal long and short axis views). The interventricular septum thickness, posterior 


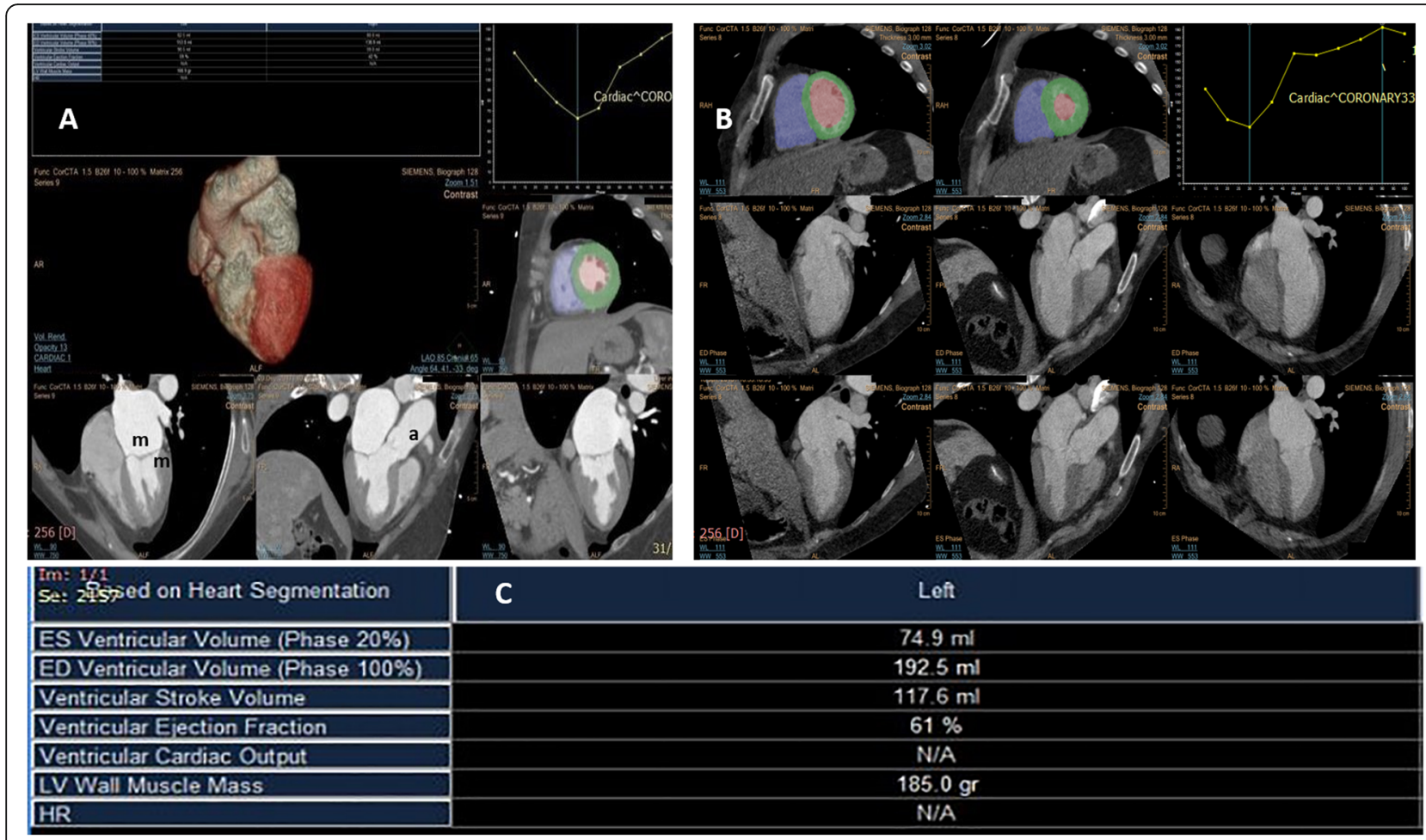

Fig. 1 a Short axis segmentation and longitudinal planes of the heart by cardiac CT. b The upper row shows short-axis view during diastole and systole. Middle row shows two-chamber view, three-chamber view, and four-chamber view during diastole. Lower row shows a two-chamber view, three-chamber view, and four-chamber view during systole C-MDCT display table containing LV functional measurements

wall thickness, and internal diameter of the left ventricle were assessed by M-mode in left parasternal short-axis images. This was done during systole and diastole) at the level just below the margin of the opened mitral valve leaflets (Fig. 2). The papillary muscles were included in the cavity during manual tracing around the endocardial borders. This was done by using an apical four-chamber image. After that, the calculation of the EF and myocardial mass was automatically performed using those data. The ESV and EDV were calculated according to the modified Simpson's method (which implies measurement by tracing endocardial border in both apical four-chamber and two-chamber views in endsystole and end-diastole [5].

\section{Statistical analysis}

The mean EF, ESV, EDV, and myocardial mass were used for statistical analysis. Using the paired two-tailed Student's $t$ test the data of ESV, EDV, EF, and myocardial mass were expressed as mean $\pm \mathrm{SD}$ and compared. Agreement for the LV volumes and function by MDCT and echocardiography were determined by Pearson's correlation coefficient for linear regression and BlandAltman analysis. The 95\% limits of agreement were defined as the range of values \pm 2 SDs from the mean value of the differences. A $p$ value $<0.05$ was considered statistically significant. To determine inter-observer agreement, intra-class correlation coefficients were used as indicators of reproducibility.

\section{Results}

This study included 50 patients with suspected cardiac problems; 27 of them were male (54\%) and 23 were female (46\%); their clinical characteristics were listed in Table 1.

\section{LVEF}

The EF obtained with the MDCT was $61.22 \pm 9.5 \%$, which was slightly higher than that obtained by echocardiography (61.14 $\pm 10.9 \%)$ with no statistical significance (Table 2). Evaluation of LVEF by linear regression analysis demonstrated a moderate correlation between MDCT and 2D echocardiography as $r=0.345$ and $p$ value $<0.05$ (Fig. 3), also Bland-Altman plot showed good inter-technique agreement analysis as it showed a mean value of difference $( \pm$ SD) of $0.8 \pm 11.6 \%$ $(p<0.05)$ between MDCT and 2D echocardiography. The $95 \%$ limits of agreement ranged from -3.3 to $3.2 \%$ (Fig. 4). 

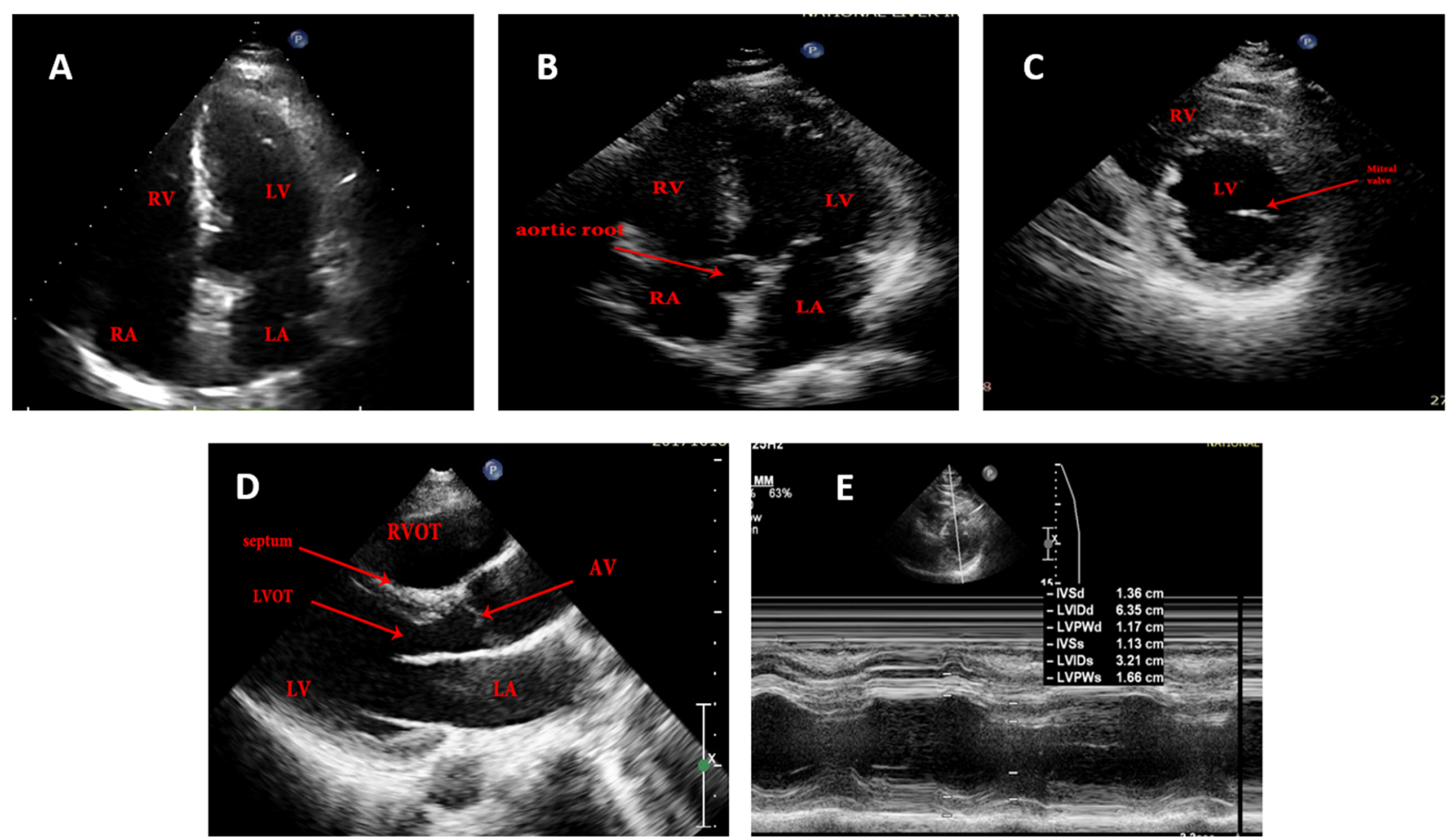

Fig. 2 Echocardiography technique. a 2D echocardiography; apical 4-chamber view. b 2D echocardiography; apical 5-chamber view. c 2D echocardiography; parasternal short-axis view. d 2D echocardiography; parasternal long-axis view. e 2D echocardiography short-axis left parasternal view of the heart with M mode measurements of the interventricular septal thickness, left ventricular diameter, and left ventricular posterior wall thickness during systole and diastole (RV, right ventricle; LV, left ventricle; RA, right atrium; LA, left atrium; RVOT, right ventricular outflow tract; LVOT, left ventricular outflow tract; AV, aortic valve; IVSd, interventricular septum thickness at end-diastole; LVIDd, left ventricular internal diameter in diastole; LVPWd, left ventricular posterior wall thickness at end-diastolic; IVSs, interventricular septum thickness at end-systole; LVIDs, left ventricular internal diameter at end-systole; LVPWs, left ventricular posterior wall thickness at end-systole)

\section{LVESV, LVEDV, and left ventricular mass}

The differences between the two modalities are summarized in Table 2 with statistical significance found only between the two modalities in left ventricular mass.

\section{Agreement assessment LVESV}

Figure 5 shows a good correlation between MDCT and 2D echocardiography $r=0.8$. $P$ value $<0.05$ with Bland Altman plot showing good inter-technique agreement as it showed a mean value of difference $( \pm$ SD) of $2.4 \pm$ $47.4 \mathrm{~mL}(p<0.05)$ between the two modalities with the $95 \%$ limits of agreement ranged from -11.2 to 16.2 .

Table 1 Clinical characteristics of the study population

\begin{tabular}{ll}
\hline Heart rate & $60-65$ \\
Hypertension & 5 \\
Diabetes mellitus & 4 \\
Coronary artery stent & 1 \\
CABG & 1 \\
Previous myocardial infarction & 0 \\
\hline
\end{tabular}

\section{LVEDV}

Figure 6 shows a good correlation between MDCT and 2D echocardiography $r=0.84, p$ value $<0.05$. BlandAltman plot also, showed good inter-technique agreement as it showed a mean value of difference $( \pm \mathrm{SD})$ of $2.28 \pm 80.4 \mathrm{~mL}(p<0.05)$ between MDCT and 2D echocardiography. The $95 \%$ limits of agreement ranged from -20.2 to 25.2 .

A negative correlation was found between EF, and both LVEDV and LVESV measured by MDCT as $p$ value $<0.05$, and no correlation between EF and LV mass. No correlation was found between EF and LVEDV, LVESV, LV mass measured by $2 \mathrm{D}$ echocardiography as $p$ value was $>0.05$ (Table 3 ).

\section{Discussion}

The functional parameters of the heart are routinely calculated by echocardiography as it is available, rapid, and noninvasive procedure. Since it is a realtime imaging technique, it is not limited by arrhythmias. But, poor acoustic windows may be produced by patient factors, like, obesity, previous operations (especially those of the cardiothoracic nature) and 
Table 2 Comparison between MDCT and 2D echocardiography regarding LV ejection fraction, LV end-systolic volume, LV enddiastolic volume, and LV mass

\begin{tabular}{|c|c|c|c|c|c|c|c|c|}
\hline & \multicolumn{3}{|l|}{ MDCT } & \multicolumn{3}{|c|}{ 2D echocardiography } & \multirow[t]{2}{*}{$t$ test } & \multirow[t]{2}{*}{$p$ value } \\
\hline & Minimum & Maximum & Mean \pm SD & Minimum & Maximum & Mean \pm SD & & \\
\hline LVEF & $30 \%$ & $77 \%$ & $61.22 \pm 9.50 \%$ & $35 \%$ & $72 \%$ & $61.14 \pm 10.90 \%$ & 0.039 & 0.969 \\
\hline LVESV & 22 & 255 & $70.23 \pm 32.35$ & 35 & 180 & $72.13 \pm 32.69$ & 0.346 & 0.730 \\
\hline LVEDV & 63.7 & 454.4 & $172.22 \pm 53.57$ & 80.24 & 371.2 & $173.76 \pm 62.45$ & 0.196 & 0.845 \\
\hline LV mass & 58.9 & 328.7 & $164.63 \pm 52.57$ & 63 & 328 & $198.32 \pm 72.54$ & 2.636 & $0.010^{*}$ \\
\hline
\end{tabular}

advanced pulmonary disease. This will prevent good delineation of cardiovascular structures. In addition, it is operator dependent $[6,7]$.

Now cardiac magnetic resonance imaging (CMRI) is considered the gold standard for noninvasive assessment of LV functional parameters, as it provides high-quality images of cardiac chambers. On the other hand, it is an expensive imaging technique with limited availability and needs proper training. In addition, accidentally motion artifacts that occur during imaging may affect the image quality. CMRI also cannot deal with patients with metallic implants [6, 7].

The assessment of coronary artery disease by cardiac CT angiography using multi-detector CT has improved a lot recently. In addition, MDCT is capable of measuring LV volumes and function with the same dose of contrast, the same amount of radiation exposure, and the same data set used for evaluation of coronary artery disease [8].
In this study, we found that mean EF obtained with MDCT was $61.22 \pm 9.50 \%$ slightly higher than that obtained by echocardiography which was $61.14 \pm 10.90 \%$. Evaluation of LVEF by linear regression analysis showed moderate correlation as $r=0.345$ and $p$ value $<0.05$, also Bland-Altman plot showed good inter-technique agreement analysis as it showed a mean value of difference $( \pm$ SD) of $0.08 \pm 11.6 \%(p<0.05)$. The $95 \%$ limits of agreement ranged from -3.3 to $3.2 \%$.

We observed that results made by MDCT are slightly higher values for LVEF when compared with 2D echocardiography, although mild reduction is expected in beta blocked patients. Although it was not statistically significant, may be the cause is limitation of evaluation technique leading to underestimation or overestimation. Mean difference in EF measurements between MDCT and 2D echocardiography is small; however, standard deviation of the mean difference is quietly high, causing wide limits of agreement. May be due to calculation of

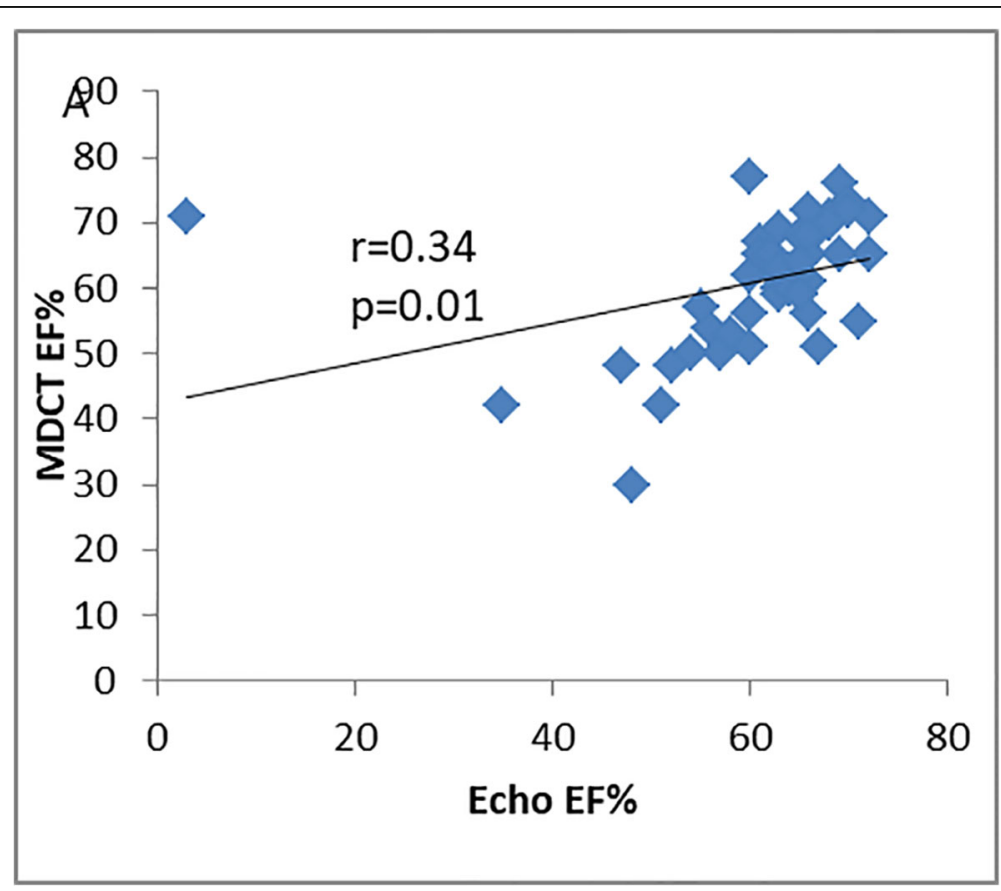

Fig. 3 Linear regression plot comparison between MDCT and 2D echocardiography assessment of LVEF. A positive correlation between LVEF as measured by MDCT and 2D echocardiography $(r=0.34, p=0.01)$ 


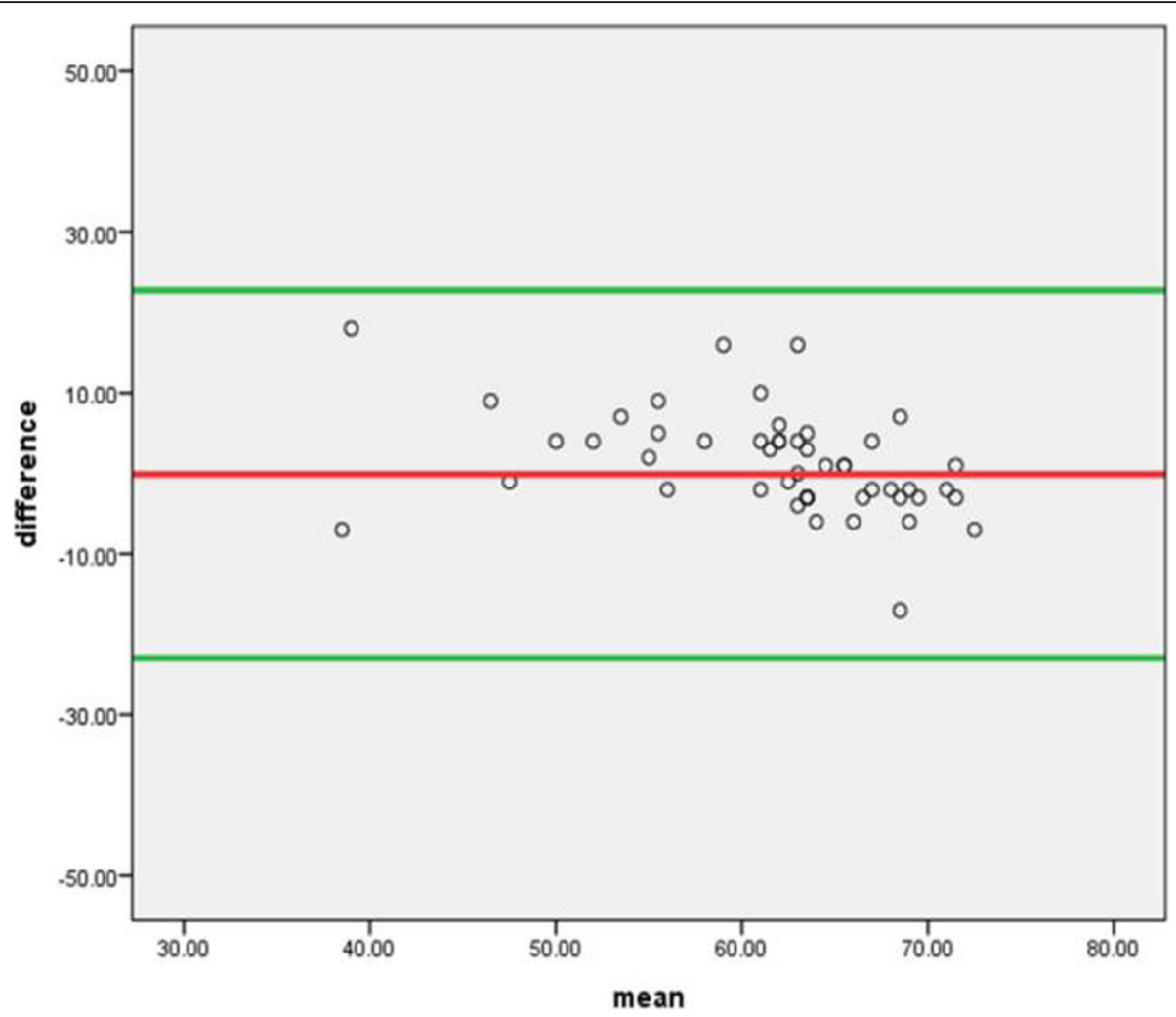

Fig. 4 Bland-Altman plot of LVEF shows the difference between EF by MDCT and 2D echocardiography plotted against the average value of them (solid red line, mean value of difference; green line, mean value of differences \pm 2 SDs) a mean value of difference $( \pm S D)$ of $0.8 \pm 11.6 \%(p<0.05)$ between MDCT and 2Dechocardiography. The $95 \%$ limits of agreement ranged from -3.3 to $3.2 \%$
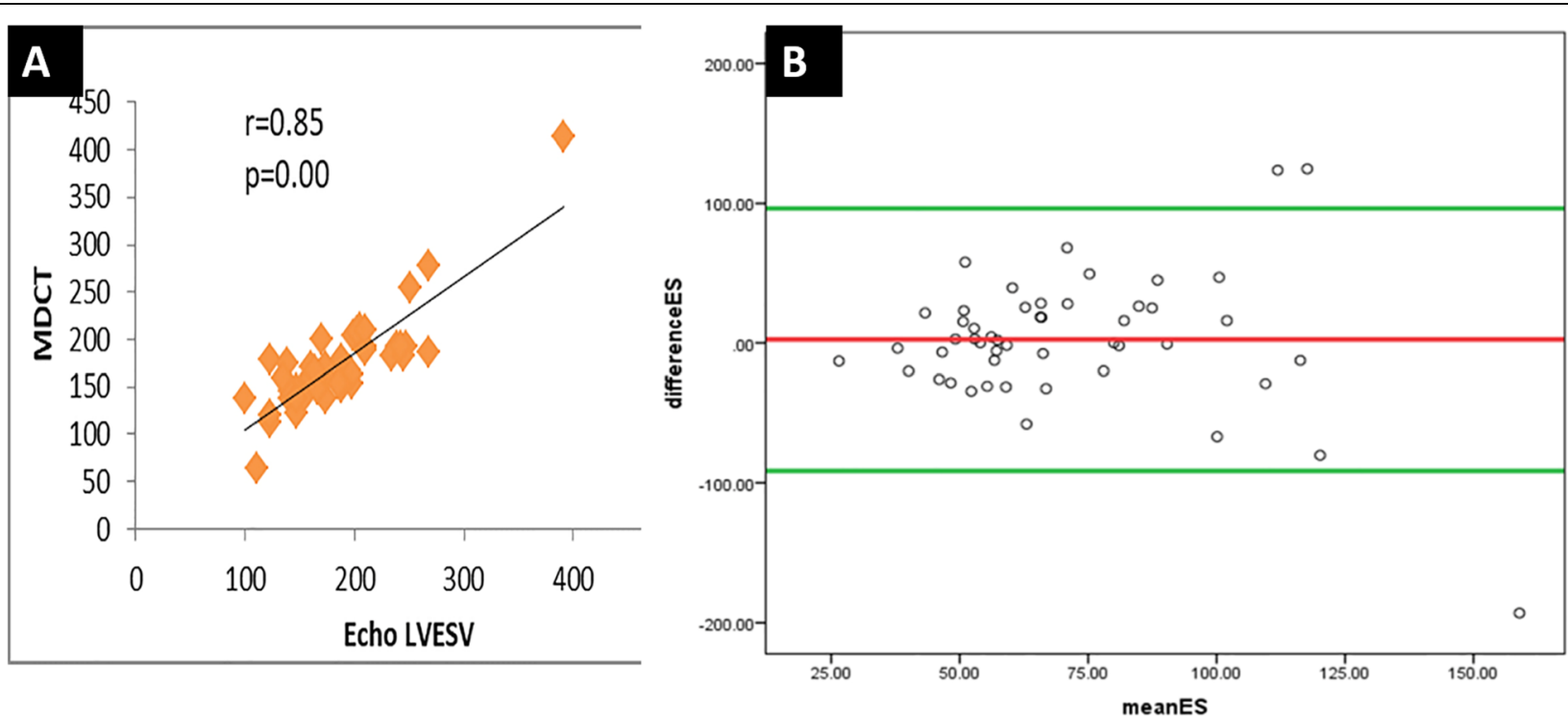

Fig. 5 a Linear regression plot correlation. b Bland-Altman plot of LVESV by MDCT and 2D echocardiography plotted against the average value of them (solid red line, mean value of difference; green line, mean value of differences \pm 2 SDs) 

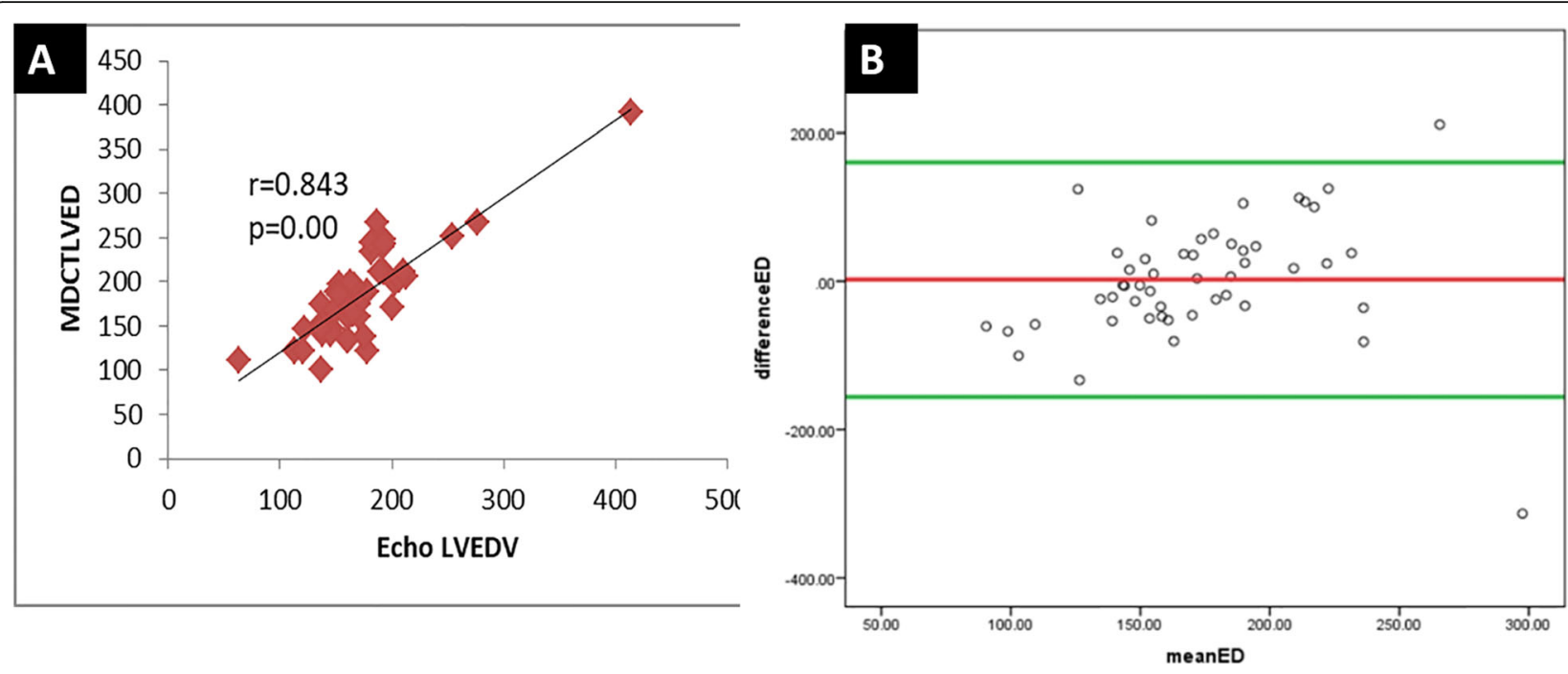

Fig. 6 a Linear regression plot correlation. b Bland-Altman plot of LVEDV as measured by MDCT and 2D echocardiography (solid red line, mean value of difference; green line, mean value of differences \pm 2 SDs)

the EF by 2D echocardiography was done using Simpson's method based on geometrical assumption.

The results of the current study are in-line with the results of previous studies that found a good correlation between MDCT and 2D echocardiography in the assessment of EF. Darpan Bansal et al. found a moderate correlation between MDCT and 2D echocardiography in 52 patients $(r=0.32, p<0.001)$ [9]. Salm et al. performed on 25 patients revealed good agreement between 16-row MDCT and echocardiography $(r=0.96 ; p<0.0001)$ [10]. Henneman et al. [11] found excellent correlation between 64-row MDCT and echocardiography in 40 patients $(r=0.91, p<0.0001)$ [10]. Kim et al. studied 19 patient with suspected CAD using 16-row MDCT and detected good correlation in the calculation of LVEF between the two modalities $(r=0.846 ; p<0.05)$ [12], and this was consistent with the current results.

The results we obtained correlate with the previous studies and confirms that assessment of LVEF is reliable with the MDCT is feasible and may be considered as a useful clinical index, compared to results made by $2 \mathrm{D}$ echocardiography. In addition, fully automated software made by $\mathrm{CT}$ proved to be faster, accurate, and user friendly.

Table 3 Correlation between ejection fraction, LVEDV, LVESV, and LV mass measured by MDCT and 2D echocardiography

\begin{tabular}{llllll}
\hline EF & MDCT & & & \multicolumn{2}{l}{ 2D echocardiography } \\
\cline { 2 - 3 } \cline { 6 - 7 } & $R$ & $p$ value & & $R$ & $p$ value \\
\hline LVEDV & -0.430 & $0.002^{*}$ & & 0.046 & 0.750 \\
LVESV & -0.703 & $0.000^{*}$ & & 0.047 & 0.748 \\
LV mass & -0.199 & 0.165 & & -0.079 & 0.583 \\
\hline
\end{tabular}

*Statistically significant ( $p$ value $<0.05$ )
In this study, we found mean LVESV measured by MDCT was $70.23 \pm 38.35$ slightly lower than that obtained by 2D echocardiography which was $72.13 \pm 32.69$ Evaluation of LVESV by linear regression analysis revealed good correlation $r=0.8, p$ value $<0.05$. BlandAltman plot showed good inter-technique agreement as it showed a mean value of difference $( \pm$ SD) of $2.4 \pm$ $47.4 \mathrm{~mL}(p<0.05)$. The $95 \%$ limits of agreement ranged from -11.2 to 16.2. Mean LVEDV measured by MDCT was $172.22 \pm 53.57$ slightly lower than that obtained by 2D echocardiography which was $173.76 \pm 62.45$. Evaluation of LVEDV by linear regression analysis revealed good correlation $r=0.84, p$ value $<0.05$. Bland-Altman plot showed good inter-technique agreement as it showed a mean value of difference $( \pm$ SD) of $2.28 \pm 80.4$ $\mathrm{mL}(p<0.05)$. The $95 \%$ limits of agreement ranged from -20.2 to 25.2 ; mean LV mass by MDCT was $164.63 \pm$ 52.57 , lower than that obtained by $2 \mathrm{D}$ echocardiography which was $198.32 \pm 72.54$.

In this study, we found that EDV and ESV obtained by MDCT are slightly lower than those calculated by 2D echocardiography. The LV volume overestimation or underestimation may be due to inclusion or exclusion of the papillary muscle [13].

In this study, the slight underestimation of LV volumes by MDCT was observed compared with 2D echocardiography; this is explained by the fact that calculation of the LV volumes measured by $2 \mathrm{D}$ echocardiography include papillary muscles but in CT papillary muscles were automatically excluded from the blood pool, which allows for precise determination of blood volume in the LV. This also explains why the mean LV mass by MDCT is lower than 2D echocardiography. 
The results of this study are in-line with results of previous studies found good correlation between 2D echocardiography and MDCT in estimation of global LV volumes. In a study by Mohamed I, Amin et al. correlation between MDCT and 2D echocardiography was excellent regarding LVESV $(r 2=0.94, p<0.001 *)$, LVEDV $(r 2=0.99, p<0.001 *)$ [8]. In the study by Graaf et al. [14] excellent correlations were observed between MDCT and 2D echocardiography for LVEDV $(r 2=0.91$; $p<0.001)$ and LVESV $(r 2=0.94 ; p<0.001)$ [13] in Lim SJ et al. [7], correlation coefficients between the two modalities for the assessment of LVESV was good $(r 2=$ 0.97, $p<0.001)$ and LVEDV was good $(r 2=0.82, p<$ 0.001 ). This in-line agreement with the current study results

We also found a negative correlation between EF and ESV; EDV measured by MDCT ( $p$ value $<0.05)$ value but no correlation between those measured by 2D echocardiography. This proves that MDCT is more reliable than 2D echocardiography in assessment of LV parameters. This is explained as $2 \mathrm{D}$ echocardiography is operator dependent.

\section{Study limitations}

Although assessment of cardiac function is feasible with 128-row MDCT, several limitations were found. The main limitation of the current study was the absence of a true gold standard such as cardiac MRI. The gold standard in the noninvasive analysis of LV function is cardiac MRI. It provides a high degree of accuracy as well as excellent temporal and spatial resolution. Concerning quantitative measurements, cardiac MRI is considered as a clinically accepted standard. In addition, MRI technique is the most relevant cardiac imaging modality available due to good contrast found between blood-filled ventricles and the surrounding myocardium; many previous studies have demonstrated excellent correlations between MDCT and MRI in the measurement of $\mathrm{LV}$ volumes and function. So, in order to validate the performance of 128-row MDCT for the assessment of LV function and volumes, a direct comparison must be done between 128-row MDCT and MRI [14, 15].

Also, in patients with a heart rate $>65 \mathrm{bpm}$, additional beta-blocking medication was administered before MDCT had been done, but not before 2D echocardiography. So potential bias may have been found by the administration of beta-blockade immediately before the MDCT examination. But new developments in MDCT technology is allowing examination of patients with higher heart rates and reducing the dose of betablockers [16, 17].

As well as the use of contrast agents in MDCT may affect LV volumes and LVEF. Another disadvantage of MDCT in general is the radiation exposure to the patient. But, assessment of LV functional parameters could be calculated retrospectively from the data acquired from the CT angiography $[14,18]$.

\section{Conclusions}

In conclusion, the current study showed that the evaluation of LV functional parameters by CT angiography is reliable. It is reasonable to utilize MDCT alone to assess LV function in clinical patients already made CT angiography examination as they do not take more radiation dose as this process is made by software and does not need another examination.

\section{Abbreviations \\ MDCT: Multi-detector computed tomography; 2D: Two dimensional; EF: Ejection fraction; ESV: End-systolic volume; EDV: End-diastolic volume; LV: Left ventricle; LV: Mass left ventricular mass; CCTA: Cardiac computed tomography angiography; CAD: Coronary artery disease}

\section{Acknowledgements \\ None}

\section{Authors' contributions}

All authors have read and approved the manuscript. M.E.AS. 1. Substantial contribution to the conception of the study. 2. Substantial contribution to the design of the study. 3. Substantial contribution to the acquisition, analysis of the data. 4. Substantial contribution to the interpretation of data. 5. Substantial contribution to the creation of the final work. 6. Substantial contribution to the study revision. 7. Substantial contribution to the accuracy or integrity of the submitted manuscript. A.A.Z 1. Substantial contribution to the conception of the study. 2. Substantial contribution to the design of the study. 3. Substantial contribution to the acquisition, analysis of the data. 4. Substantial contribution to the interpretation of data. 5. Substantial contribution to the creation of the final work. 6. Substantial contribution to the study revision. 7. Substantial contribution to the accuracy or integrity of the submitted manuscript. A.M.AA.A. 1. Substantial contribution to the conception of the study. 2. Substantial contribution to the design of the study. 3. Substantial contribution to the acquisition, analysis of the data. 4. Substantial contribution to the interpretation of data. 5. Substantial contribution to the creation of the final work. 6. Substantial contribution to the study revision. 7. Substantial contribution to the accuracy or integrity of the submitted manuscript. S.AH.H. 1. Substantial contribution to the conception of the study. 2. Substantial contribution to the design of the study. 3. Substantial contribution to the acquisition, analysis of the data. 4. Substantial contribution to the interpretation of data. 5. Substantial contribution to the creation of the final work. 6 . Substantial contribution to the study revision. 7. Substantial contribution to the accuracy or integrity of the submitted manuscript.

\section{Funding}

Not applicable

\section{Availability of data and materials}

The datasets used and/or analyzed during the current study are available from the corresponding author on reasonable request.

\section{Ethics approval and consent to participate}

Our study was approved by the Institutional Research Board (IRB)/ Institutional Ethics Committee (IEC) following the Helsinki Declaration with informed written consent obtained from every subject prior to the examination after full explanation of the technique. Faculty of Medicine Menoufia University Ref. No. 2018/3/6/703.

Consent for publication

All authors gave consent to publish the manuscript.

Competing interests

The authors declare that they have no competing interests. 


\section{Author details}

Radiodiagnosis Department, National Liver Institute, Menoufia University, Menoufia, Egypt. ${ }^{2}$ Radiodiagnosis Department, Faculty of Medicine, Menoufia University, Menoufia, Egypt.

Received: 28 February 2020 Accepted: 12 May 2020

Published online: 26 May 2020

\section{References}

1. Han R, Sun K, Lu B et al (2017) Diagnostic accuracy of coronary CT angiography combined with dual-energy myocardial perfusion imaging for detection of myocardial infarction. Exp Ther Med 14:207-213

2. Oe H, Watanabe N, Miyoshi T, Osawa K, Akagi T, Kanazawa S, Ito H (2018) Potential benefit of a simultaneous, side-by-side display of contrast MDCT and echocardiography over routine sequential imaging for assessment of adult congenital heart disease: a preliminary study. J Cardiol 72(5):395-402

3. Rumberger JA (2016) Assessment of cardiac structure and function by computed tomography angiography. In: Budoff MJ, Shinbane JS (eds) Cardiac CT imaging: diagnosis of cardiovascular disease, 3rd edn. Springer International Publishing, pp 191-210

4. Iannaccone M, Gili S, De Filippo O et al (2019) Diagnostic accuracy of functional, imaging and biochemical tests for patients presenting with chest pain to the emergency department: a systematic review and meta-analysis. Eur Heart J Acute Cardiovasc Care 8(5):412-420

5. Kosaraju A, Makaryus AN. Left Ventricular Ejection Fraction. [Updated 2020 Feb 11]. In: StatPearls. Treasure Island (FL): StatPearls Publishing; 2020. Available from: https:/www.ncbinlm.nih.gov/books/NBK459131/

6. Singh RM, Singh BM, Mehta JL (2014) Role of cardiac CTA in estimating left ventricular volumes and ejection fractions. World J Radiol 6(9):669-676

7. Lim SJ, Choo KS, Park YH et al (2011) Assessment of left ventricular function and volume in patients undergoing 128-slice coronary $\subset T$ angiography with ECGbased maximum tube current modulation: a comparison with echocardiography. Korean J Radiol 12:156-162 https:/doi.org/10.3348/kir.2011.12.2.156

8. Amin MI, Hassan HA, Mousa MIA (2018) Utility of 128-row multidetector CT in quantitative evaluation of global left ventricular function in patients with coronary artery disease. EJRNM 49:12-22

9. Bansal D, Singh R, Sarkar M et al (2008) Assessment of left ventricular function: comparison of cardiac multidetector-row computed tomography with two-dimension standard echocardiography for assessment of left ventricular function. Int I Cardiovasc Imaging

10. Salm LP, Schuijf JD, de Roos A et al (2006) Global and regional left ventricular function assessment with 16-detector row CT: comparison with echocardiography and cardiovascular magnetic resonance. Eur J Echocardiogr 7:308-314

11. Henneman MM, Schuijf JD, Jukema JW et al (2006) Assessment of global and regional left ventricular function and volumes with 64-slice MSCT: a comparison with 2D echocardiography. J Nuclear Cardiol 13(4):480-487

12. Kim TH, Hur J, Kim SJ et al (2005) Two-phase reconstruction for the assessment of left ventricular volume and function using retrospective ECG-gated MDCT: comparison with echocardiography. Am J Roentgenol 185(2):319-325.

13. Mo YH, Jaw FS, Wang YC et al (2011) Effects of propranolol on the left ventricular volume of normal subjects during $C T$ coronary angiography. Korean J Radiol 12:319-326

14. Graaf FR, Schuijf J et al (2010) Assessment of global left ventricular function and volumes with 320-row multidetector computed tomography: a comparison with 2D-echocardiography. J Nucl Cardiol 17(2):225-231

15. Hegde S, Bhat V, Gadabanahalli K, Kuppuswamy M (2014) MDCT derived left ventricular function in relation to echocardiography: validation and revising the role with the evolving technology. J Cardiovasc Echography 24(1):18-24

16. Schlosser T, Mohrs OK, Magedanz A et al (2007) Assessment of left ventricular function and mass in patients undergoing computed tomography (CT) coronary angiography using 64-detector-row CT: comparison to magnetic resonance imaging. Acta Radiol 48:30-35

17. Krishnam MS, Tomasian A, Iv M, Ruehm SG et al (2008) Left ventricular ejection fraction using 64-slice $\mathrm{CT}$ coronary angiography and new evaluation software: initial experience. Br J Radiol 81:450-455

18. Malahfji M, Al-Mallah MH (2019) Cardiac CT assessment of right and left ventricular and valvular function. Curr Cardiovasc Imaging Rep 12(6):23

\section{Publisher's Note}

Springer Nature remains neutral with regard to jurisdictional claims in published maps and institutional affiliations.

\section{Submit your manuscript to a SpringerOpen ${ }^{\circ}$ journal and benefit from:}

- Convenient online submission

- Rigorous peer review

- Open access: articles freely available online

- High visibility within the field

- Retaining the copyright to your article

Submit your next manuscript at $\boldsymbol{\nabla}$ springeropen.com 